\title{
Influencia del entorno institucional en el desarrollo del emprendimiento español. Un análisis empírico
}

\author{
Gómez Haro, Samuel* \\ Salmerón Gómez, Román**
}

\begin{abstract}
Resumen
La débil capacidad de emprendimiento actual en las empresas españolas se muestra como uno de los factores que imposibilitan la recuperación económica. Los estudios relacionan esta debilidad con el entramado institucional existente, pero son requeridos análisis más complejos en este ámbito. El presente trabajo responde a la necesidad de crear modelos estadísticos consistentes que analicen la relación entre el entorno institucional y orientación emprendedora. Para el análisis, se utiliza un cuestionario personal con directores generales de 150 empresas españolas contestado telefónicamente. Los datos obtenidos fueron analizados a través de análisis factorial y de regresión lineal. Los resultados muestran que el entorno institucional ejerce diferentes influencias sobre la orientación emprendedora, lo que repercute en el nivel de emprendimiento de las empresas españolas.
\end{abstract}

Palabras clave: Entorno institucional, orientación emprendedora, empresa española.

Recibido: 07-01-11. Aceptado: 17-05-11

* Departamento de Organización de Empresas, Universidad de Granada, Campus Universitario de La Cartuja s/n, 18071 Granada (España). E-mail: samugh@ugr.es

** Departamento de Métodos Cuantitativos para la Economía y la Empresa, Universidad de Granada, Campus Universitario de La Cartuja s/n, 18071 Granada (España).

E-mail: romansg@ugr.es

Los autores desean agradecer a los proyectos de investigación del Ministerio de Ciencia e Innovación (ECO2010-20483) y de Excelencia de la Junta de Andalucía (P08-SEJ-04057) la provisión de fondos para la investigación. También agradecemos a los editores de Revista Venezolana de Gerencia la posibilidad de publicar en la revista y a los tres revisores anónimos por sus sugerencias y comentarios en el proceso de evaluación. 
Influencia del entorno institucional en el desarrollo del emprendimiento español...

Gómez Haro, Samuel y Salmerón Gómez, Román

\title{
Influence of the Institutional Environment on the Development of Spanish Entrepreneurship. An Empirical Analysis
}

\begin{abstract}
The weak capacity of current entrepreneurship in Spanish companies is indicated as one of the factors that prevents economic recovery. Studies relate this weakness with the existing institutional framework, but more complex analyses are required in this area. This work responds to the need for creating consistent statistical models that analyze the relationship between the institutional environment and entrepreneurial orientation. For this analysis, data were obtained from CEOs of 150 Spanish companies using a personal questionnaire answered by phone. Data were analyzed by factor analysis and linear regression. Results show that the institutional environment exerts different influences on entrepreneurial orientation, which affects the level of entrepreneurship in Spanish companies.
\end{abstract}

Key words: Institutional environment, entrepreneurial orientation, Spanish firms.

\section{Introducción}

El comportamiento emprendedor es un aspecto vital para el crecimiento, la rentabilidad y la supervivencia de las organizaciones (Bosma et al. , 2009; Bosma y Levie, 2010). Las organizaciones se orientan hacia esta postura con base a una serie de procesos, prácticas y actividades que posibilitan dicha actividad emprendedora, medido generalmente en términos de proactividad, innovación y asunción de riesgos (Covin y Slevin, 1991; Green et al., 2008; Miller, 1983).

En el desarrollo de la orientación emprendedora en las organizaciones y sus miembros influyen diferentes factores, donde el entorno institucional ha sido generalmente excluido de este análisis (Covin y Slevin, 1991; Knight, 1997; Zahra, 1993). La importancia que adquiere en tiempos recientes el entorno institucional muestra la oportunidad de analizar cómo éste presiona e influye en las organizaciones para que adopten posturas más emprendedoras (Baumol et al., 2009; Bruton et al., 2010).

El entorno institucional se define en base a la existencia de una serie de reglas y requisitos a los que deben someterse las organizaciones si desean recibir apoyo y legitimidad (Scott y Meyer, 1991). Este entorno institucional debe ser tenido en cuenta en los modelos que analizan la orientación emprendedora de las empresas, ya que es un factor con una gran influencia en la gestión de las organizaciones, al proporcionar estabilidad y significado al comportamiento social (Scott, 1995). De hecho, los trabajos de investigación que relacionan entorno institucional y emprendimiento han despertado gran atención en los tiempos recientes (Lim et al., 2010; Urbano-Pulido et al., 2007; Vaillant y Lafuente, 2007), aunque es necesario un mayor contraste empírico que facilite la creación de modelos explicativos entre este entorno institucional y las diferentes manifestaciones del emprendimiento (Bruton et al., 2010; Spencer y Gómez, 2004). 
Este trabajo analiza cómo el entorno institucional, a través de sus dimensiones reguladoras, normativas y cognitivas, influye en la orientación emprendedora de las empresas. Para ello, el trabajo se centra en el caso de las empresas españolas, siendo uno de los países del marco de la Unión Europea (UE) que está sufriendo los problemas de la actual crisis económica. Recientemente, los investigadores están analizando el modelo del emprendimiento español incluyendo al entorno institucional, pero estos trabajos son fragmentados o descriptivos en sus resultados, por lo que en el presente trabajo se utiliza un modelo de regresión lineal que de una mayor consistencia empírica a los resultados de la investigación obtenidos.

Para ello, se utiliza una muestra con datos obtenidos a través de un cuestionario respondido por directores generales de 150 empresas españolas, y a través del análisis factorial y técnicas de regresión lineal se analiza cómo están influyendo los distintos factores institucionales en el desarrollo de una orientación emprendedora efectiva en las empresas. El presente trabajo cumple con la necesidad demandada por los investigadores (Bruton et al., 2010; Spencer y Gómez, 2004) de crear modelos estadísticos consistentes que analicen la relación entre el entorno institucional y orientación emprendedora.

Para conseguir el objetivo del trabajo, se analizan los constructos de orientación emprendedora y entorno institucional en primer lugar. Posteriormente, se analiza el caso de la empresa española para establecer las hipótesis de investigación. A continuación, se muestran los resultados obtenidos a través de un análisis factorial y de regresión. Por último, se finaliza con las conclusiones del trabajo, así como las limitaciones y las líneas futuras de investigación.

\section{La orientación emprendedora}

La orientación emprendedora se define como el conjunto de procesos, prácticas y actividades relacionadas con la toma de decisiones que posibilitan la actividad emprendedora (Dess y Lumpkin, 2005; Covin y Slevin, 1991; Lumpkin y Dess, 1996; Miller, 1983) y puede ser un instrumento para lograr el éxito empresarial, debido a que es un aspecto vital para el crecimiento, la rentabilidad y la supervivencia de las organizaciones, siendo considerado el motor de la economía moderna y del desarrollo social a través del crecimiento económico, la generación de empleo y el fomento de la innovación (Bosma et al., 2009; Bosma y Levie, 2010; Zahra, 1995).

Su importancia viene determinada porque numerosas investigaciones previas han verificado de forma empírica que la orientación emprendedora de las empresas está relacionada de forma positiva con la rentabilidad y el crecimiento de la empresa (Covin et al., 2006; Covin y Slevin, 1991; Ireland et al., 2009; Wiklund y Shepherd, 2005).

Investigaciones anteriores conceptualizan la orientación emprendedora como un constructo con varias dimensiones (Dess y Lumpkin, 2005; Kreiser et al., 2002; Lumpkin y Dess, 1996; Miller, 1983). Aunque existen diferentes modelos, está ampliamente aceptado en investigación que la orientación emprendedora se basa 
Influencia del entorno institucional en el desarrollo del emprendimiento español... Gómez Haro, Samuel y Salmerón Gómez, Román

en la capacidad de innovación, proactividad y asunción de riesgos (Barringer y Bluedorn, 1999; Green et al., 2008; Kreiser y Justin, 2010; Miller, 1983).

La innovación se relaciona con el compromiso de la organización a gestionar y apoyar nuevas ideas y procesos que tengan como consecuencia nuevos productos, servicios o procesos. La proactividad se traduce en la búsqueda de oportunidades para introducir nuevos productos o servicios en el mercado, actuando anticipadamente ante nuevas demandas y de forma competitiva. Por último, la asunción de riesgos consiste en el grado en el que los directivos están dispuestos a comprometer un mayor número de recursos con un incierto retorno (Dess y Lumpkin, 2005).

Las primeras investigaciones sobre orientación emprendedora apuntan que las empresas emprendedoras asumen un nivel de riesgo mayor que el resto de empresas, especialmente cuando se encuentran en situaciones de gran incertidumbre (Antoncic y Hisrich, 2004; Mintzberg, 1988; Zahra, 1993). Posteriormente, los investigadores operativizaron el comportamiento emprendedor como el conjunto de innovaciones de producto-mercado, proactividad en la toma de decisiones, y la capacidad de asumir riesgos (Covin et al., 2006; Kreiser et al., 2002; Miller, 1983; Miller y Friesen, 1978). Actualmente, el nivel de emprendimiento exhibido por la empresa se basa en la combinación de cada una de las sub-dimensiones, ya que una empresa es realmente emprendedora, si exhibe niveles altos de innovación, riesgo y proactividad (Covin y Slevin 1991, Green et al., 2008).
En el desarrollo de la orientación emprendedora en las organizaciones, y sus miembros, influyen diferentes factores, entre los que el entorno tiene un papel clave (Covin y Slevin, 1991; Kreiser y Justin, 2010; Zahra, 1993). Sin embargo, normalmente el análisis del entorno se ha realizado a través de las dimensiones más clásicas (hostilidad, dinamismo) obviando la existencia de un entorno institucional que influye igualmente en la gestión organizativa y en su nivel de emprendimiento (Baumol et al., 2009; Bruton et al., 2010). La importancia que adquiere en tiempos recientes el entorno institucional muestra la oportunidad de analizar cómo éste presiona e influye en las organizaciones para que adopten posturas más emprendedoras.

No obstante, el peso de las instituciones nacionales y comunitarias en este aspecto es cada vez mayor, por lo cual existe una fuerte tendencia en diseñar, por parte de las entidades públicas, medidas para incentivar el emprendimiento, el clima emprendedor y la creación de una sociedad más innovadora y creativa que aproveche las oportunidades existentes en el mercado (European Comission, 2003, 2004). Por ello, el análisis desde una perspectiva institucional es fundamental y necesario para la mejor comprensión del fenómeno emprendedor en sí mismo (Baumol et al., 2009; Bruton et al., 2010; Spencer y Gómez, 2004).

\section{La influencia del entorno institucional}

La teoría institucional centra su estudio en los aspectos del contexto en el que están inmersas las organizaciones, 
haciendo hincapié en el conjunto de valores, normas o creencias que actúan como mitos racionales guiando el comportamiento de las organizaciones (Meyer y Rowan, 1977). Esta teoría es útil para el entendimiento de las relaciones sociales que establece la organización con otras organizaciones y con las instituciones con las que coexiste, señalando como factores de éxito la conformidad con las reglas y normas institucionales (DiMaggio y Powell, 1983; Meyer y Rowan, 1977). Asimismo, la teoría institucional ha demostrado ser un marco teórico idóneo para el análisis organizativo (DiMaggio y Powell, 1991).

Scott (1995) analiza el entorno institucional en base a tres dimensiones: cognitivas, normativas y reguladoras; que proporcionan estabilidad y significado al comportamiento social. Kostova (1999) interpreta las dimensiones institucionales de Scott y plantea a través del concepto "Country Institutional Profile" (CIP) la relación de las diferentes dimensiones con la gestión organizativa.

La dimensión reguladora del entorno institucional incluye las políticas gubernamentales que proveen apoyo a los nuevos negocios y facilitan los esfuerzos de los emprendedores. La dimensión cognitiva se refiere al conocimiento y habilidades que poseen los habitantes de un determinado país, respecto a temas relacionados con la gestión empresarial, convirtiendo esos conocimientos en esquemas cognitivos compartidos. Por último, la dimensión normativa se refiere al grado en el que los ciudadanos de un país concreto valoran la mentalidad creativa e innovadora de las personas y las organizaciones que lo componen (Busenitz et al., 2000).
Con base a lo expuesto, desde un punto de vista institucional, para un mayor desarrollo del emprendimiento es necesario que las instituciones mejoren determinados elementos estructurales, institucionalizando un entorno donde la capacidad para asumir riesgos, la innovación e iniciativa en la gestión organizativa sean elementos estructurales comunes en todas las organizaciones del campo. Con base a esta premisa, se considera que la existencia de un entorno institucional que fomente el emprendimiento influye positivamente en la capacidad emprendedora de sus organizaciones y ciudadanos.

\section{El caso de la empresa española. Modelo de estudio}

Los trabajos de investigación que relacionan entorno institucional y emprendimiento han atraído mucha atención en los tiempos recientes (Amine y Staub, 2009; Lim et al., 2010; Vaillant y Lafuente, 2007). Sin embargo, los investigadores reclaman mayor evidencia empírica que facilite la creación de modelos explicativos entre las distintas dimensiones institucionales y manifestaciones del emprendimiento (Bruton et al., 2010; Spencer y Gómez, 2004).

Esta necesidad de profundizar en el tema se debe a que la mayoría de los análisis sobre la influencia del entorno institucional en el emprendimiento han sido descriptivos y fragmentados (Stephen et al., 2005; Urbano et al., 2007).

La actual situación económica demanda empresas con iniciativas y creatividad, ya que la orientación emprendedora es la forma de dotar a las economías desarrolladas de una mayor capacidad 
Influencia del entorno institucional en el desarrollo del emprendimiento español... Gómez Haro, Samuel y Salmerón Gómez, Román

competitiva (European Comission, 2003, 2004), y en el caso de España está necesidad es más acentuada. Actualmente, España cuenta con tasa de desempleo del $20,5 \%$ frente al $8,5 \%$ de la media de los países pertenecientes a la Organización para la Cooperación y el Desarrollo Económicos - OCDE (OECD Harmonised Unemployment Rates, 2010). Aunque muchos factores influyen en esta situación, diferentes expertos y organismos nacionales vienen subrayando los problemas estructurales que presenta el país, relacionados con la debilidad de su capacidad de emprendimiento, situándola en las últimas posiciones en las clasificaciones elaboradas por la Comisión Europea sobre los índices de actividad y clima emprendedor en los países europeos (De la Vega et al., 2009).

Concretamente, en el presente trabajo se analiza cómo el entorno institucional está afectando al comportamiento emprendedor de las empresas españolas.

Por un lado, el emprendimiento encuentra barreras a su desarrollo, resultantes de un entramado de reglamentos y leyes que lo convierte en un factor que inhibe la asunción de riesgos, no sólo en el caso de la creación de nuevos negocios, sino también en la expansión de los ya existentes, así como en la innovación de sus fórmulas organizativas. La presencia de regulaciones en mercados clave, como el laboral o de desarrollo de los mercados financieros, provocan una rigidez que desincentiva a las personas y empresas a la asunción de iniciativas y nuevos riesgos empresariales (Capelleras et al., 2008; Documentos Círculo de Empresarios, 2009), además de la pre- sencia de onerosas cargas administrativas (Grilo e Irigoyen, 2006).

Esto es precisamente lo que indican los datos: son muchos quienes declaran en encuestas que desearían ser empresarios, pero muy pocos los que lo son. Año tras año los informes señalan que una de las barreras al desarrollo del emprendimiento en España, es la ausencia o la presencia insuficiente, de políticas de apoyo públicas para el otorgamiento de facilidades de financiamiento a las personas y empresas que quieren ser emprendedoras (de la Vega, 2010). Por lo que el marco regulador, relacionado con leyes y reglas, se muestra como un limitador del comportamiento emprendedor. En función de los argumentos anteriores, se establece la primera hipótesis de investigación que relaciona la influencia de la dimensión reguladora y la orientación emprendedora.

Hipótesis 1: La dimensión reguladora del entorno institucional influye negativamente en la orientación emprendedora de las empresas españolas.

Por otro lado, los análisis establecen que una de las peores condiciones existentes es la referente a la educación y a la formación emprendedora, especialmente en edad escolar (de la Vega et al., 2010). La sociedad española muestra un relativo desconocimiento de las cuestiones relacionas con el emprendimiento y la gestión empresarial (Valdaliso-Gago, 2005). Este desconocimiento parte de un sistema educativo, tanto en educación secundaria (estudiantes cuya edad oscila entre12-16 años) como bachillerato (estudiantes entre 16-18 años), donde el tratamiento de la figura del empresario y de 
la actividad económica y empresarial no ha sido realizado con el rigor y la profundidad necesaria (González, 2003).

Esto incluye también al ámbito universitario, donde tampoco se promueve el desarrollo de actividades empresariales y el conocimiento de aspectos relacionados con el mundo empresarial y su gestión. La ausencia de una educación empresarial objetiva evita la creación de esquemas cognitivos compartidos comunes sobre temas empresariales, lo que conlleva una mayor aversión al riesgo y un temor a tomar iniciativas innovadoras, es decir, una menor orientación emprendedora.

Por tanto, el marco cognitivo, relacionado con la existencia de esquemas de conocimiento compartidos sobre gestión empresarial, se muestra actualmente como un limitador del comportamiento emprendedor de las empresas españolas. En función de los argumentos anteriores, se establece la segunda hipótesis de investigación que relaciona la influencia de la dimensión cognitiva y la orientación emprendedora.

Hipótesis 2: La dimensión cognitiva del entorno institucional influye negativamente en la orientación emprendedora de las empresas españolas.

Por último, los valores y la cultura de una sociedad influyen en la orientación de las personas hacia el emprendimiento, pudiendo lograr que se valore positivamente la creatividad y la puesta en práctica de ideas en actividades empresariales. Una sociedad donde exista una mentalidad abierta, con iniciativa y que valore de forma positiva la creatividad y la innovación, crea un entorno normativo que alienta a las organizaciones a cam- biar los modelos de orientación estratégica hacia posiciones emprendedoras (Ahlstrom y Bruton, 2002; Nguyen et al., 2009; Kshetri, 2009).

En España, la figura del empresario ha sido vista históricamente con cierto recelo y temor, lo que ha hecho que el país haya ocupado los últimos lugares en las clasificaciones de percepción social del empresariado. Pero estos análisis muestran también que, en los últimos veinte años, se ha fortalecido la imagen positiva de las empresas y de sus actividades, reconociéndolas como fuente generadoras de riqueza y desarrollo (Valdaliso-Gago, 2005), por lo que se propone que el marco normativo, relacionado con los valores y la cultura, si favorece actualmente el comportamiento emprendedor de las empresas españolas. En función de los argumentos anteriores, se establece la tercera hipótesis de investigación que relaciona la influencia de la dimensión normativa del entorno institucional y la orientación emprendedora.

Hipótesis 3: La dimensión normativa del entorno institucional influye positivamente en la orientación emprendedora de las empresas españolas.

En la Figura 1 se observa el modelo de investigación resultante del análisis teórico y del establecimiento de hipótesis que se analiza en el siguiente epígrafe.

\section{Estudio estadístico}

Para conseguir los objetivos del estudio y confirmar las hipótesis planteadas en la sección anterior, a continuación se aplicarán métodos multivariables para analizar de forma simultánea, conjuntos amplios de variables y captar la informa- 
Influencia del entorno institucional en el desarrollo del emprendimiento español... Gómez Haro, Samuel y Salmerón Gómez, Román

Figura 1. Modelo de investigación

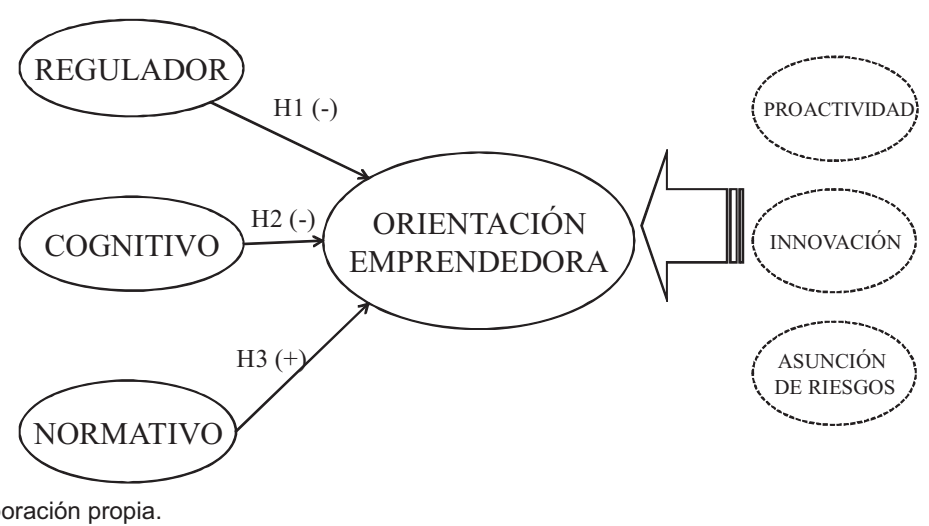

Fuente: Elaboración propia.

ción presentes en las mismas. Más concretamente, se pondrán en práctica las técnicas de análisis factorial y regresión uniecuacional múltiple.

El análisis factorial es una técnica que tiene por objeto, como bien es sabido, explicar un conjunto de variables observadas por un pequeño número de variables latentes (no observadas) que reciben el nombre de factores. Mientras que el análisis de regresión consiste en investigar la relación estadística que existe entre una variable dependiente y una o más variables independientes. Para poder realizar esta investigación, se debe postular una relación funcional entre las variables. Debido a su simplicidad analítica, la forma funcional que más se utiliza en la práctica es la relación lineal.

Por tanto, a continuación se realizará un análisis factorial en busca de los factores anteriormente indicados de orientación emprendedora y de las tres dimensiones del entorno institucional: reguladora, normativa y cognitiva. Es decir, el objetivo del análisis factorial será el de explicar el total de las variables conside- radas a partir de los cuatro factores anteriormente indicados. En segundo lugar, el análisis de regresión será usado para analizar la influencia de los factores cognitivo, normativo y regulador sobre la orientación emprendedora. Es decir, será la técnica que permita confirmar o descartar las hipótesis planteadas.

Para la realización del cuestionario, se adaptan distintos constructos de ítems de la literatura emprendedora e institucional (Busenitz et al., 2000; Knight, 1997; Zahra, 1993), quedando un cuestionario, que sería enviado a los directores generales de las empresas, formado por las preguntas de la Tabla 1 del anexo (adviértase que en negrita se resalta el nombre con el se codificará cada pregunta en el posterior análisis estadístico).

Todas las preguntas se midieron con escalas de tipo likert de siete puntos, lo que permite el uso de procedimientos estadísticos que se pueden aplicar a escalas de intervalos, ya que se está suponiendo que existe un continuo detrás de las mismas. Por ejemplo, haciendo preguntas sobre la medida en que está de 
acuerdo o no con una afirmación y acotando las respuestas a 1-7 ó 1-5, se supone que detrás de esa respuesta existe un continuo del que se está extrayendo puntos clave que permiten medir algo que en sí no tiene medida, como es la percepción.

La muestra contaba con más de 6000 empresas de la base de datos Duns \& Bradstreet, de las que se escogieron 1455 mediante un muestreo aleatorio simple para realizar el cuestionario por teléfono a sus directores generales. Del total de empresas contactadas, se obtuvieron 150 cuestionarios contestados de forma completa $(10,30 \%)$, que son las que constituyen la muestra analizada. Se consideró importante utilizar sólo cuestionarios respondidos de forma completa en todas sus preguntas para evitar la aparición de datos perdidos que pudiesen influir en la calidad del posterior análisis estadístico. Para más detalles sobre la muestra consultar el Cuadro 1.

Mediante el análisis factorial, tal y como se ha indicado, se pretende simplificar las múltiples y complejas relaciones que puedan existir entre el conjunto de variables consideradas, mediante el menor número de factores no directamente observables que expliquen suficientemente las variables estudiadas (en este caso las preguntas del cuestionario), perdiendo el mínimo de información y de forma que sean fácilmente interpretables. Para la realización del análisis se empleó el programa estadístico SPSS, en su versión 19.

Para conseguir con éxito los requisitos anteriores, es necesario un alto grado de correlación entre las variables observadas, dado que entonces éste puede deberse a la existencia de variables no observables directamente por el modelo (factores). Luego, antes de realizar el análisis factorial hay que plantearse estudiar si las variables consideradas están correlacionadas entre sí, ya que en caso negativo, no tendría sentido aplicar dicha técnica.

Con tal objetivo se realiza la prueba de esfericidad de Barlett, que contrasta la hipótesis nula de que la matriz de correlación poblacional sea igual a la identidad,

\section{Cuadro 1. Ficha técnica}

\begin{tabular}{ll}
\hline Sector & Empresas españolas \\
Tipo & Cuestionario realizado vía telefónica \\
Población objetivo & 6884 empresa \\
Número de empresas contactadas & 1455 empresas \\
Tamaño muestra & 150 empresa \\
Tasa de respuesta & $10.3 \%$ \\
Error muestral & $7.91 \%$ \\
Nivel de significación & $95 \%, \mathrm{p}=\mathrm{q}=0.5, \mathrm{z}=1.96$ \\
Encuesta dirigida a & Directores Generales \\
Fecha trabajo campo & Abril-Mayo de 2009 \\
\hline
\end{tabular}

Fuente: Elaboración propia. 
Influencia del entorno institucional en el desarrollo del emprendimiento español... Gómez Haro, Samuel y Salmerón Gómez, Román

es decir, no hay correlación entre las variables observadas. En este caso, puesto que se obtiene un $p$-valor menor que $10^{-3}$, se rechaza claramente la hipótesis nula. Por otro lado, Kaiser-Meyer y Olkin (1970) definieron la medida KMO para la adecuación muestral global al modelo factorial, basada en los coeficientes de correlación observados de cada par de variables y en sus coeficientes de correlación parcial. En el caso de que exista adecuación de los datos, a un modelo de análisis factorial, la medida KMO será próxima a la unidad. Evidentemente, cuanto más próximos a 1 estén los valores de dicha prueba, mejor será la adecuación de los datos a un modelo factorial. Puesto que en este caso se obtiene un valor de 0.88 para la prueba $\mathrm{KMO}$, se puede afirmar que la adecuación de los datos a un modelo factorial es muy buena.

Una vez justificada la idoneidad de realizar un análisis factorial sobre las variables consideradas (Cuadro 2), al existir una correlación significativa entre las variables, en la Tabla 1 se muestran las comunalidades iniciales (siempre iguales a 1) y finales. Dichas comunalidades ex- presan el porcentaje de varianza que se ha explicado de cada una de las variables mediante el análisis factorial. Como se observa, al estar todas (excepto una) por encima de 0.5 se consideran satisfactorias.

El siguiente paso será el de analizar el número de factores a considerar. En este caso, la literatura analizada indica la idoneidad de considerar 4 factores (ver Figura 1), tres factores que indican cada una de las dimensiones del entorno institucional: regulador, normativo y cognitivo, y un factor único que analice la orientación emprendedora de la empresa. En la Tabla 2 se muestra el porcentaje de variabilidad total explicada por dichos factores.

Como puede observarse, el primer componente explica un $36.857 \%$ de la varianza total explicada, la segunda un $14.039 \%$, la tercera un $8.774 \%$ y la cuarta un $7.42 \%$, para un total de un $67.09 \%$ de la varianza total. Puesto que en ciencias sociales es normal considerar extraer un número de factores que explique entre un $60 \%-65 \%$ de la varianza total, se considerará como buena esta elección del nú-

Tabla 1. Comunalidades

\begin{tabular}{ccccccccc}
\hline Variable & Inicial & Final & Variable & Inicial & Final & Variable & Inicial & Final \\
\hline VAR1 & 1 & $\mathbf{0 . 7 2 8}$ & VAR9 & 1 & $\mathbf{0 . 6 5 5}$ & VAR17 & 1 & $\mathbf{0 . 6 7 8}$ \\
VAR2 & 1 & $\mathbf{0 . 7 3 0}$ & VAR10 & 1 & $\mathbf{0 . 6 6 0}$ & VAR18 & 1 & $\mathbf{0 . 7 1 3}$ \\
VAR3 & 1 & $\mathbf{0 . 7 5 9}$ & VAR11 & 1 & $\mathbf{0 . 6 2 2}$ & VAR19 & 1 & $\mathbf{0 . 7 6 6}$ \\
VAR4 & 1 & $\mathbf{0 . 7 2 6}$ & VAR12 & 1 & $\mathbf{0 . 6 4 6}$ & VAR20 & 1 & $\mathbf{0 . 7 0 7}$ \\
VAR5 & 1 & $\mathbf{0 . 7 9 8}$ & VAR13 & 1 & $\mathbf{0 . 6 4 9}$ & VAR21 & 1 & $\mathbf{0 . 7 6 7}$ \\
VAR6 & 1 & $\mathbf{0 . 8 3 8}$ & VAR14 & 1 & $\mathbf{0 . 5 6 3}$ & VAR22 & 1 & $\mathbf{0 . 6 1 1}$ \\
VAR7 & 1 & $\mathbf{0 . 7 9 2}$ & VAR15 & 1 & $\mathbf{0 . 5 2 5}$ & VAR23 & 1 & $\mathbf{0 . 5 0 3}$ \\
VAR8 & 1 & $\mathbf{0 . 4 5 5}$ & VAR16 & 1 & $\mathbf{0 . 6 0 6}$ & VAR24 & 1 & $\mathbf{0 . 6 0 5}$ \\
\hline
\end{tabular}

Fuente: SPSS. 


\section{Cuadro 2. Variables}

\begin{tabular}{|c|c|}
\hline Codificación & Pregunta \\
\hline VAR1 & $\begin{array}{l}\text { Las instituciones públicas de este país ayudan a las empresas a realizar nueva activida- } \\
\text { des empresariales. }\end{array}$ \\
\hline VAR2 & $\begin{array}{l}\text { Los organismos gubernamentales ofrecen fondos públicos suficientes para nuevas activi- } \\
\text { dades de negocio de las empresas. }\end{array}$ \\
\hline VAR3 & $\begin{array}{l}\text { Las instituciones nacional y local apoyan preferentemente a las empresas que quieren } \\
\text { realizar nuevas actividades empresariales. }\end{array}$ \\
\hline VAR4 & $\begin{array}{l}\text { Incluso después de fracasar en la actividad empresarial, las instituciones estimulan a las } \\
\text { empresas que intentan poner en marcha nuevas actividades empresariales. }\end{array}$ \\
\hline VAR5 & Las empresas saben cómo hacer frente a situaciones de riesgo. \\
\hline VAR6 & Las empresas saben cómo gestionar el riesgo. \\
\hline VAR7 & $\begin{array}{l}\text { Las empresas saben dónde encontrar información para el desarrollo de nuevas activida- } \\
\text { des empresariales. }\end{array}$ \\
\hline VAR8 & En este país, el pensamiento innovador y creativo es visto como una ruta hacia el éxito. \\
\hline VAR9 & Los emprendedores son personas admiradas en nuestro país. \\
\hline VAR10 & La sociedad admira a aquellas organizaciones que son emprendedoras. \\
\hline VAR11 & $\begin{array}{l}\text { En general, la dirección de la empresa es propensa a aceptar proyectos de elevado ries- } \\
\text { go. }\end{array}$ \\
\hline VAR12 & $\begin{array}{l}\text { Cuando la empresa se enfrenta a situaciones de toma de decisiones que implican incerti- } \\
\text { dumbre, normalmente se adopta una postura atrevida y agresiva que permita maximizar } \\
\text { la probabilidad de explotar el potencial de oportunidades. }\end{array}$ \\
\hline VAR13 & $\begin{array}{l}\text { En general, la dirección de su empresa cree que, dada la naturaleza del entorno, es nece- } \\
\text { sario actuar con valentía y atrevimiento para lograr los objetivos de la organización. }\end{array}$ \\
\hline VAR14 & En general, su empresa suele tomar la iniciativa en los procesos de negociación. \\
\hline VAR15 & $\begin{array}{l}\text { Comparado con los competidores, es frecuente que su empresa sea la primera en introdu- } \\
\text { cir nuevas herramientas en su gestión (productos/servicios, técnicas administrativas, tec- } \\
\text { nologías, etc.). }\end{array}$ \\
\hline VAR16 & $\begin{array}{l}\text { Comparado con los competidores, la empresa adopta una postura de mercado muy com- } \\
\text { petitiva. }\end{array}$ \\
\hline VAR17 & El énfasis de la organización en el desarrollo de nuevos productos o servicios. \\
\hline VAR18 & El ratio de introducción de nuevos productos o servicios en el mercado. \\
\hline VAR19 & El gasto de la organización en actividades de desarrollo de nuevos productos o servicios. \\
\hline VAR20 & El número de nuevos productos introducidos por la organización ya existentes en el mercado \\
\hline VAR21 & El número de nuevos productos introducidos por la organización ya existente en el mercado. \\
\hline VAR22 & $\begin{array}{l}\text { El porcentaje de ingresos de la organización generados por productos que no existían } \\
\text { hace tres años. }\end{array}$ \\
\hline VAR23 & El número de cambios drásticos en las líneas de productos o servicios. \\
\hline VAR24 & El énfasis de la dirección en I+D, liderazgo tecnológico e innovación. \\
\hline
\end{tabular}

Fuente: Elaboración propia. 
Influencia del entorno institucional en el desarrollo del emprendimiento español... Gómez Haro, Samuel y Salmerón Gómez, Román

Tabla 2. Varianza total explicada

\begin{tabular}{cccc}
\hline Factor & Total & $\begin{array}{c}\% \\
\text { Varianza }\end{array}$ & $\begin{array}{c}\% \\
\text { Acumulado }\end{array}$ \\
\hline 1 & 8.846 & 36.857 & 36.857 \\
2 & 3.369 & 14.039 & 50.896 \\
3 & 2.106 & 8.774 & 59.670 \\
4 & 1.781 & 7.420 & 67.090 \\
\hline
\end{tabular}

Fuente: SPSS.

mero de factores, que además cumple los principios de interpretabilidad (como se verá posteriormente son fácilmente interpretables) y de parsimonia (menor número de factores posibles) exigibles a todo análisis factorial.

Para la estructura factorial considerada, se analiza matriz de componentes rotados por el método Varimax. Adviértase que en la selección anterior se han usado las cargas factoriales, que son el medio para interpretar la función que cada variable desempeña para definir cada factor. Las cargas con valor absoluto igual o superior a 0.5 son significativas, ya que explican un mínimo del $25 \%$ de la varianza del factor. Por tanto, en los cuatro factores anteriores se han considerado aquellas variables con una carga factorial mínima de 0.5 .

Los resultados muestran que desde VAR11 a VAR24 las variables cargan en el factor 1 con cargas factoriales que están entre 0.656 y 0.850 . El grupo de variables desde VAR1 a VAR4 cargan en el segundo factor con valores desde 0.812 a 0.837 . Las variables VAR5 a VAR8 se agrupan en el tercer factor con cargas de 0.853 a 0.872 . Por último, en el cuarto factor se agrupan las variables VAR8 a VAR10, con cargas entre 0.545 y 0.759 .
Una vez que la estructura factorial ha quedado clara, el último paso será interpretar cada uno de los factores desde el punto de vista de la literatura existente.

El primer factor, formado por las variables 11 a la 24 , trata sobre los distintos aspectos que la empresa debe incorporar en su gestión para ser emprendedora. Estas preguntas están orientadas hacia la capacidad de la empresa para asumir riesgos, de tomar iniciativas y competir de forma agresiva en el mercado (proactividad) y de su nivel de innovación. El análisis demuestra además la importancia de un constructo único que exhiba niveles importantes de las tres características para una mejor orientación emprendedora.

En el segundo factor resultante, variables 1 a la 4, vemos ítems que analizan si en el entorno institucional en el que opera la empresa existen recursos legales promovido por los distintos entes institucionales que facilitan su actividad económica, por lo que se consideran explicativos del factor regulador del entorno institucional.

El tercero, formado por las variables 5 a 7 , muestra si por norma general la sociedad muestra un conocimiento sobre temas empresariales y aspectos relacionados como la gestión y el riesgo. Este conocimiento crea unos esquemas compartidos por la sociedad, por lo que se relacionan con el factor cognitivo del entorno institucional.

En el cuarto factor, los ítems 8 a 10 tratan sobre si la sociedad valora de forma positiva aspectos como la innovación, la creatividad o la iniciativa empresarial. Se identifica este factor como el factor normativo del entorno institucional. 
Asimismo, siguiendo las indicaciones planteadas en la sección 4 , a continuación se estudiará si los factores cognitivos, reguladores y normativos influyen en la orientación emprendedora y en qué sentido. Es decir, se van a estudiar desde un punto de vista empírico las hipótesis formuladas en dicha sección.

Con tal objetivo se analiza el modelo uniecuacional múltiple dado por:

$$
Y t=\alpha \cdot X_{1}+\beta \cdot X_{2}+\gamma \cdot X_{3}+u t,
$$

donde; $Y$ denota al factor de orientación emprendedora, $X_{1}$ al factor regulador, $X_{2}$ al factor cognitivo y $X_{3}$ al normativo. En tal caso, los coeficientes de las variables explicativas denotan la magnitud del efecto que las mismas tienen sobre la variable explicada. Además, $u$ denota la perturbación aleatoria del modelo que se supone centrada, homocedástica, incorrelada y distribuida según la ley normal.

A partir de los valores de los factores sobre los elementos de la muestra observados (puntuaciones factoriales), del análisis factorial anterior, se ha obtenido la siguiente estimación:

$$
\begin{gathered}
\hat{Y}_{t}=-0.103 \cdot X_{1}-0.189 \cdot X_{2}+0.107 \cdot X_{3}, \\
(0.0843) \quad(0.0034) \quad(0.0655)
\end{gathered}
$$

donde; entre paréntesis se indica el p-valor asociado al contraste de significación individual a cada coeficiente. De esta forma, se obtiene que el factor cognitivo es significativo al $5 \%$, influyendo de forma negativa sobre la orientación emprendedora, por lo que se verifica la H2. Mientras que los factores regulador y normativo lo son al $1 \%$ de significación. En tal caso, el factor regulador influirá negativamente también sobre la actividad emprendedora, verificando la $\mathrm{H} 1$; mientras que el normativo lo hace positivamente, verificando la H3.

Adviértase que el modelo original incumplía la hipótesis básica de heteroscedasticidad necesaria en todo análisis de regresión lineal (para el contraste de White se obtenía un p-valor de $2.3172 \cdot 10^{-6}$, por lo que se rechazaba claramente la hipótesis nula que el modelo era homocedástico). De forma que esta estimación se ha realizado bajo la existencia de heteroscedasticidad $\mathrm{y}$, por tanto, corrigiéndola.

Para dar validez al modelo $\mathrm{y}$, por tanto, a las afirmaciones anteriores, se recurre a la prueba ANOVA que estudia la significación conjunta de los parámetros estimados. Puesto que se obtiene un pvalor de 0.000141 , se rechaza la hipótesis nula de que todos los coeficientes son nulos de forma simultánea y se puede afirmar que hay algún tipo de asociación entre las variables dependientes y la independiente.

Es importante destacar que aunque se ha obtenido un coeficiente de determinación bajo, 0.129077, teniendo en cuenta la equivalencia existente entre el coeficiente de determinación y el test ANOVA, dicho coeficiente de determinación es significativo. Es decir, a partir de la relación anteriormente indicada, se obtiene que el modelo es explicativo si,

$$
R^{2}>\frac{\frac{k-1}{n-k} \cdot F_{k-1, n-k}(1-\alpha)}{1+\frac{k-1}{n-k} \cdot F_{k-1, n-k}(1-\alpha)}=R_{s i g}^{2}
$$


Influencia del entorno institucional en el desarrollo del emprendimiento español... Gómez Haro, Samuel y Salmerón Gómez, Román

siendo $k$ el número de variables presentes en el modelo, $n$ el número de observaciones de que se disponen y $\mathrm{F}_{\mathrm{k}-1, \mathrm{n}-\mathrm{k}}(1-\alpha)$ el punto de una distribución $\mathrm{F}$ de Snedecor con $k-1$ y $n$ - $k$ grados de libertad que deja a su izquierda una probabilidad 1- $\alpha$. Puesto que en este caso $R_{\text {sig }}^{2}=0.03996865$, es evidente que, tal y como se ha adelantado, el coeficiente de determinación obtenido implica que el modelo es explicativo.

Finalmente, tras realizar la prueba de normalidad de Kolmogorov-Smirnov sobre los residuos del modelo, se obtiene que éstos se distribuyen normalmente a un $1 \%$ de significación ( $p$-valor asociado de 0.023). La comprobación de esta hipótesis es fundamental, ya que toda la inferencia realizada en el modelo uniecuacional múltiple se basa en el supuesto de normalidad de los residuos.

\section{Conclusiones}

El emprendimiento ha demostrado ser una de las opciones más viables para el desarrollo económico y social de los últimos tiempos, por lo que cada vez recibe una mayor atención por parte de los investigadores que analizan su funcionamiento, sus consecuencias y la forma de estimularlo para un desarrollo efectivo. La teoría institucional sirve de sustento para tratar de desarrollar cómo las empresas que se encuentran en un marco económico determinado pueden estimular su comportamiento emprendedor, ya que el entorno institucional provee las reglas a seguir por las organizaciones para poder obtener legitimidad en sus acciones.

La contribución principal del presente trabajo es la demostración empírica de cómo el entorno institucional, influ- ye actualmente en el nivel de emprendimiento de la empresa española, medido a través de su capacidad de innovación, proactividad y asunción de riesgos (orientación emprendedora). Hasta la fecha los informes y trabajos que analizaban el emprendimiento desde una perspectiva institucional eran fragmentados o descriptivos en sus análisis, por lo que era necesario un trabajo empírico más complejo que analizase dicha relación.

Este trabajo subraya la importancia de las diferentes dimensiones del entorno institucional en su influencia en el comportamiento emprendedor de las empresas. Además de la influencia del entorno legal y regulador, la existencia de una cultura que aliente la iniciativa y la innovación y una base de esquemas cognitivos compartidos relativos a la gestión empresarial influye en la orientación emprendedora de las empresas y, por tanto, en la puesta en funcionamiento de nuevas iniciativas empresariales.

Los resultados confirman que el marco regulador, compuesto por las reglas y los programas de apoyo a las actividades empresariales, resulta un obstáculo a la orientación emprendedora de las empresas, debido especialmente a la existencia de fuertes regulaciones y el complejo entramado burocrático para la creación y puesta en marcha de nuevas iniciativas empresariales. El marco cognitivo también influye negativamente en la orientación emprendedora de la empresa, ya que se tiene déficit de conocimiento empresarial en la sociedad española. Este déficit no crea un ámbito cognitivo común donde se comparta planteamientos y conocimientos de temas de empresa, limitando la orientación emprendedora. 
Por último, pese a que históricamente la percepción social del emprendedor no ha sido la más adecuada, los resultados muestran que actualmente el marco normativo, compuesto por la cultura y la valoración que hace la sociedad del empresario y de las empresas con iniciativa y creatividad, influye de forma positiva en la orientación emprendedora de las organizaciones. Esto confirma la tendencia de una sociedad española que si bien históricamente estaba en las últimas posiciones sobre la percepción positiva del empresario y la actividad económica, los análisis descriptivos recientes mostraban un cambio de tendencia en esta percepción (Círculo de Empresarios, 2009; Valdaliso-Gago, 2005).

El reto actual de la empresa española, de las instituciones y de la sociedad española, en general, es el cambio de paradigma en su postura ante el emprendimiento. Desde la perspectiva institucional, este cambio debe originarse mediante la existencia de leyes, reglamentos y programas de apoyo empresarial que faciliten la puesta en práctica de nuevas actividades corporativas y que la sociedad esté compuesta por personas con iniciativa, capaces de asumir riesgos, creativas e innovadoras, ya que es una de las vías de éxito empresarial. Por ello, una mayor formación empresarial en todos los niveles educativos sería imprescindible para lograr el cambio social referido. Esta orientación del entorno institucional, el cambio de actitud respecto a esta materia de las organizaciones y la mayor agresividad y competitividad en las posturas empresariales de los ciudadanos en general, logrará potenciar la capacidad emprendedora de la sociedad en su conjunto, algo que redundará en dicha capacidad a nivel organizacional.

El trabajo presenta una serie de limitaciones: un solo informante, la naturaleza transversal del trabajo y los resultados parten de una muestra de empresas españolas. No obstante, la literatura institucional avisa de la dificultad de utilizar muestras agregadas de diferentes países, debido a los distintos entornos institucionales existentes y la dificultad de establecer un único entorno institucional común que generalice los resultados (Busenitz et al., 2000).

Los futuros trabajos empíricos deben superar las limitaciones expuestas con anterioridad, profundizando y ampliando los resultados aquí obtenidos. Sería de gran interés analizar las mismas relaciones en organizaciones de diferentes países para establecer comparaciones y, si es posible, establecer un modelo general para la literatura emprendedora. La posibilidad de ampliar el estudio empírico a ámbitos europeos, daría la oportunidad de verificar los resultados obtenidos a una escala superior, despuntando los análisis meramente descriptivos actuales y obtener modelos explicativos del fenómeno emprendedor corporativo que incluyan la influencia institucional, aspecto que ha sido obviado por los investigadores.

\section{Referencias bibliográficas}

Alshtrom, David y Bruton, Garry D. (2002). An institutional perspective on the role of culture in shaping strategic actions by technology-focused entrepreneurial forms in China. Entrepreneurship Theory and Practice, Vol. 26, No. 4, pp. 53-69. 
Influencia del entorno institucional en el desarrollo del emprendimiento español... Gómez Haro, Samuel y Salmerón Gómez, Román

Amine, Lyn S. y Staub, Karim M. (2009). Women entrepreneurs in Sub-Sharan Africa: An institutional theory analysis from a social marketing point of view. Entrepreneurship \& Regional Development, Vol. 21, No. 2, pp. 183-211.

Antoncic, Bostjan y Hisrich, Robert D. (2004). Corporate entrepreneurship contingencies and organizational wealth creation. Journal of Small Business and Enterprise Development, Vol. 23, No. 6, pp. 518-550.

Barringer, Bruce R. y Bluedorn, Allen C. (1999). The relationship between corporate entrepreneurship and strategic management. Strategic Management Journal, Vol. 20, pp. 421-444.

Baumol, William J.; Litan, Robert E. y Schramm, Carl J. (2009). Good capitalism, bad capitalism, and the economics of growth and prosperity. New Haven, CT: Yale University Press.

Bosma, Niels y Levie, Jonathan (2010). Global Entrepreneurship Monitor. 2009. Executive Report. GEM.

Bosma, Niels; Acs, Zoltan J.; Autio, Erkko; Coduras, Alicia y Levie, Jonathan (2009). Global Entrepreneurship Monitor. 2008. Executive Report. GEM.

Bruton, Garry D.; Ahlstrom, David y Li, Han-Lin (2010). Institutional theory and entrepreneurship: Where are we now and where do we need to move in the future? Entrepreneurship Theory and Practice, Vol. 34, No.3, pp. 421-440.

Busenitz, Lowell W.; Gómez, Carolina y Spencer. Jennifer W. (2000). Country institutional profiles: Interlocking entrepreneurial phenomena. Academy of Management Journal, Vol. 43, No. 5, pp. 994-1003.

Capelleras, Joan-lluis; Mole, Kevin F.; Greene, Francis J. y Storey, David J. (2008). Do more heavely regulated economies have poorer performing new ventures? Evidence from Britain and Spain. Journal of International Business Studies, Vol. 39, pp. 688-704.

Covin, Jeffrey G.; Green, K.M. y Slevin, Dennis P. (2006). Strategic process effects on the entrepreneurial orientation-sales growth rate relationship. Entrepreneurship Theory and Practice, Vol. 30 , No. 1 , pp. $57-82$.

Covin, Jeffrey G. y Slevin, Dennis P. (1991). A conceptual model of entrepreneurship as firm behaviour. Entrepreneurship Theory and Practice, Vol. 16, No. 1, pp. 7-25.

De la Vega García-Pastor, Ignacio; Coduras, Alicia; Cruz, Cristina; Justo, Rachida y González, Isabel (2009). Global Entrepreneurship Monitor. 2008. Spain Executive Report. GEM.

De la Vega García-Pastor, Ignacio; Coduras, Alicia; Cruz, Cristina; Justo, Rachida y González, Isabel (2010). Global Entrepreneurship Monitor. 2009. Spain Executive Report. GEM.

DiMaggio, Paul J. y Powell, Walter W. (1983). The iron cage revisited: Institutional isomorphism and collective rationality. American Sociological Review, Vol. 48, pp. 147-160.

DiMaggio, Paul J. y Powell, Walter W. (1991). The New Institutionalism in Organizational Analysis, The University of Chicago Press.

Documentos Círculo de Empresarios (2009). El espíritu emprendedor: elemento esencial para afrontar la crisis económica española. http://www.circulodeempresarios.org/wp-ontent/uploads/2009/06/espititu-1.pdf.

European Commission (2003). Green Paper: Entrepreneurship in Europe. Communication from the Commission to the Council, the European Parliament, the European Economic and Social Committee and the Committee of the Regions, COM (98) 222. 
European Commission (2004). Action Plan: The European Agenda for Entrepreneurship. Communication from the Commission to the Council, the European Parliament, the European Economic and Social Committee and the Committee of the Regions, COM (03) 70 .

Green, Kimberly M.; Covin, Jeffrey G. y Slevin, Dennis P. (2008). Exploring the relationship between strategic reactiveness and entrepreneurial orientation: The role of structure-style fit. Journal of Business Venturing, Vol. 23, pp. 356-383.

González, Manuel J. (2003). El empresario y la economía de mercado, Monografía 11, junio 2003, Círculo de Empresarios.

Grilo, Isabel. e Irigoyen, Jesús-María (2006). Entrepreneurship in the EU: To Wish and Not to Be. Small Business Economics, Vol. 16, pp. 305-318.

Ireland, R. Duane; Covin, Jeffrey G. y Kuratko, Donald F. (2009). Conceptualizing corporate entrepreneurship strategy. Entrepreneurship Theory and Practice, Vol. 33, No. 1, pp. 19-46.

Knight, Garry A. (1997). Cross cultural reliability and validity of a scale to measure firm entrepreneurial orientation. Journal of Business Venturing, Vol.12, pp. 213-225.

Kostova, Tatiana (1999). Transnational transfer of strategic organizational practices: A contextual perspective. Academy of Management Review, Vol. 24, No. 2, pp. 308-324.

Kreiser, Paul M. y Davis, Justin (2010). Entrepreneurial orientation and firm performance: The unique impact of innovativeness, proactiveness, and risk-taking, Journal of Small Business and Entrepreneurship, Vol. 23, No. 1, pp. 39-51.
Kreiser, Paul M.; Marino, Louis D. y Weaver, K. Mark (2002). Assessing the psychometric properties of the entrepreneurial orientation scale: A multi-country analysis, Entrepreneurship Theory and Practice, Vol. 26, No. 4, pp. 71-94

Kshetri, Nir (2009). Entrepreneurship in post-socialist economies: A typology and institutional contexts for market entrepreneurship, Journal of International Entrepreneurship, Vol. 7, pp. 236-259.

Lim, Dominic S.K; Morse, Eric A.; Mitchell, Ronald K. y Seawright, Kristie K. (2010). Institutional environment and entrepreneurial cognitions: A comparative business perspective. Entrepreneurship Theory and Practice, Vol. 34, No. 3, pp. 491-516.

Lumpkin, G. Tom y Dess, Gregory G. (1996). Clarifying the entrepreneurial orientation construct and linking it to performance. Academy of Management Review, Vol. 21, No. 1, pp. 135-172.

Meyer, John W. y Rowan, Brian (1977). Institutionalized organizations: Formal structure as myth and ceremony. American Journal of Sociology, Vol. 83, pp. 340-363.

Miller, Danny (1983). The correlates of entrepreneurship in three types of firms. Management Science, Vol. 29, No. 7, pp. 770-791.

Miller Danny y Friesen, Peter H. (1978): Archetypes of strategy formulation. Management Science, Vol. 24, pp. 921933.

Mintzberg, Henry (1988). La estructuración de las organizaciones. Ariel S.A., Barcelona.

Nguyen, Thang V.; Bryant, Scott E.; Rose, Jerman; Tseng, Chiung-Hui y Kapasuwan, Supara (2009). Cultural values, market institutions, and entrepreneurship potential: A comparative study of the United States, Taiwan, 
Influencia del entorno institucional en el desarrollo del emprendimiento español...

Gómez Haro, Samuel y Salmerón Gómez, Román

and Vietnam. Journal of Developmental Entrepreneurship, Vol. 14, No. 1, pp. 21-37.

OCDE (2010). OECD Harmonised Unemployment Rates, August 2010, http:// www.oecd.org/dataoecd/45/21/4617 5570.pdf

Scott, W. Richard (1995). Institutions and Organizations, Sage Publications.

Scott, W. Richard y Meyer, John W. (1991). The organization of societal sectors: Propositions and early evidence. En W.W. Powell y P. DiMaggio (Eds.) The New Institutionalism in Organizational Analysis. The University of Chicago Press.

Spencer, Jennifer W. y Gómez, Carolina (2004). The relationship among national institutional structures, economics factors and domestic entrepreneurial activity: a multicountry study. Journal of Business Research, Vol. 57, pp. 1098-1107.

Stephen, Frank H.; Urbano, David y van Hemmen, Stefan (2005). The Impact of institutions on entrepreneurial activity. Managerial and Decisions Economics, Vol. 26, pp 413-419.

Urbano-Pulido, David; Díaz-Casero, Juan Carlos y Hernández-Mogollón, Ricardo (2007). Evolución y principios de la teoría económica institucional. Una propuesta de aplicación para el análi- sis de los factores condicionantes de la creación de empresas. Investigaciones Europeas de Dirección y Economía de la Empresa, Vol. 13, No. 3, pp. 183-198.

Valdaliso-Gago, Jesús-María (2005). El espíritu emprendedor en España: un análisis histórico. En Cuervo-García, J.A. y Sastre-Castillo (Eds), La empresa y el espíritu emprendedor de los jóvenes, Ministerio de Educación.

Vaillant, Yancy y Lafuente, Esteban (2007). Do different institutional frameworks condition the influence of local fear or failure and entrepreneurial examples over entrepreneurial activity? Entrepreneurship \& Regional Development, Vol. 19, No. 4, pp. 313-337.

Wiklund, Johan y Shepherd, Dean (2005). Entrepreneurial orientation and small business performance: A configurational approach. Journal of Business Venturing, Vol. 20, No. 1, pp. 71-91.

Zahra, Shaker A. (1993). A conceptual model of entrepreneurship as firm behavior: A critique and extension. Entrepreneurship Theory and Practice, Vol. 17, No. 4, pp. 5-21.

Zahra, Shaker A. (1995). Corporate entrepreneurship and financial performance: The case of management leveraged buyouts. Journal of Business Venturing, Vol. 10, pp. 225-247. 